\title{
Reversible Diffusion by Thermal Fluctuations
}

\author{
Aleksandar Donev, Thomas G. Fai and Eric Vanden-Eijnden ${ }^{1}$ \\ ${ }^{1}$ Courant Institute of Mathematical Sciences, New York University, New York, NY 10012
}

\begin{abstract}
A model for diffusion in liquids that couples the dynamics of tracer particles to a fluctuating Stokes equation for the fluid is investigated in the limit of large Schmidt number. In this limit, the concentration of tracers is shown to satisfy a closed-form stochastic advection-diffusion equation that is used to investigate the collective diffusion of hydrodynamically-correlated tracers through a combination of Eulerian and Lagrangian numerical methods. This analysis indicates that transport in liquids is quite distinct from the traditional Fickian picture of diffusion. While the ensembleaveraged concentration follows Fick's law with a diffusion coefficient that obeys the Stokes-Einstein relation, each instance of the diffusive mixing process exhibits long-ranged giant fluctuations around its average behavior. We construct a class of mesoscopic models for diffusion in liquids at different observation scales in which the renormalized diffusion coefficient depends on this scale. This indicates that the Fickian diffusion coefficient in liquids is not a material constant, but rather, changes with the scale at which experimental measurements are performed.
\end{abstract}

Diffusion is one of the most ubiquitous transport process. It is, arguably, the simplest dissipative mechanism. Fick's law of diffusion is "derived" in most elementary textbooks, and relates diffusive fluxes to the gradient of chemical potentials via a diffusion coefficient that is typically thought of as a material property. Yet, there are several hints that diffusion in liquids is, in fact, rather subtle. A first hint is that the Stokes-Einstein (SE) prediction for the diffusion coefficient involves the viscosity of the fluid, a seemingly independent transport property. This suggests a connection between momentum transport and diffusion and may explain why the SE prediction is in surprisingly reasonable agreement with measurements even in cases where it should not apply at all, such as molecular diffusion. A second hint is that nonequilibrium diffusive transport is accompanied by "giant" longrange correlated thermal fluctuations [1-3], which have been measured using light scattering and shadowgraphy techniques [2, 4, 6]. It is now well-understood that these unexpected features of diffusion in liquids stem from the contributions of advection by thermal velocity fluctuations [7-10]. It has long been appreciated in statistical mechanics and nonequilibrium thermodynamics circles that thermal fluctuations exhibit long-ranged correlations in nonequilibrium settings [1, 3. The aim of this Letter is to show that these fluctuations are also of overarching importance to transport in fluids, a fact that has not been widely recognized so far.

In either gases, liquids or solids, one can, at least in principle, coarse-grain Hamiltonian dynamics for the atoms (at the classical level) to obtain a model of diffusive mass transport at hydrodynamic scales. This procedure is greatly simplified by first coarse-graining the microscopic dynamics to a simpler stochastic description, which is done by using kinetic theory for gases or Markov jump models for diffusion in solids. In both cases the picture that emerges is that of independent Brownian walkers performing uncorrelated random walks in continuum (gases) or on a lattice (solids). By contrast, in liquids the physical picture is rather different and must account for hydrodynamic correlations among the diffusing particles. In a liquid, molecules become trapped (caged) over long periods of time, as they collide with their neighbors. Therefore, momentum and energy are exchanged (diffuse) much faster than the molecules themselves can escape their cage. The main mechanism by which molecules diffuse is the motion of the whole cage when a large-scale velocity fluctuation (coordinated motion of parcels of fluid) moves a group of molecules and shifts and rearranges the cage.

Most previous theoretical studies of molecular diffusion are based on some form of mode-mode coupling, which is essentially a perturbative analysis in the strength of the thermal fluctuations $7-[12$. In this Letter we formulate a simple model for diffusion in liquids at microscopic and mesoscopic scales and use it to make a precise assessment of the contribution of fluctuations to diffusive transport. Our model is a simplified (coarse-grained) representation of the complex molecular processes that underlie mass transport in liquids. It mimics all of the crucial features of realistic liquids, while also being tractable analytically and numerically. Through a mix of theoretical and numerical studies, we show that this model exhibits realistic physical behaviors that differ from those of standard models of Fickian diffusion via uncorrelated random walks. In particular, we find that there is an unexpected connection between flows at small and large scales, and at microscopic and mesoscopic scales diffusion in liquids resembles turbulent diffusion.

Our model describes the motion of passive tracer particles advected by thermal velocity fluctuations, and can be used to describe the dynamics of fluorescentlylabeled molecules in a Fluorescence Recovery After Photo-bleaching (FRAP) experiment, the transport of nano-colloidal particles in a nanofluid, or the motion of the molecules in a simple fluid. We will neglect direct interactions among the particles, which is appropriate when tracers are dilute. The evolution of the incompress- 
ible fluid velocity, $\boldsymbol{v}(\boldsymbol{r}, t)$ with $\boldsymbol{\nabla} \cdot \boldsymbol{v}=0$, is assumed to satisfy the linearized fluctuating Navier-Stokes equation

$$
\rho \partial_{t} \boldsymbol{v}+\boldsymbol{\nabla} \pi=\eta \boldsymbol{\nabla}^{2} \boldsymbol{v}+\sqrt{2 \eta k_{B} T} \boldsymbol{\nabla} \cdot \mathcal{W},
$$

where $\mathcal{W}(\boldsymbol{r}, t)$ denotes a white-noise symmetric tensor field (stochastic momentum flux) with covariance chosen to obey a fluctuation-dissipation principle [3],

$$
\left\langle\mathcal{W}_{i j}(\boldsymbol{r}, t) \mathcal{W}_{k l}\left(\boldsymbol{r}^{\prime}, t^{\prime}\right)\right\rangle=\left(\delta_{i k} \delta_{j l}+\delta_{i l} \delta_{j k}\right) \delta\left(t-t^{\prime}\right) \delta\left(\boldsymbol{r}-\boldsymbol{r}^{\prime}\right) \text {. }
$$

The details of the microscopic coupling between the fluid and the passive tracer are complicated [13] and some approximations are required. We will assume that the evolution of the concentration of a large collection of tracers, $c(\boldsymbol{r}, t)$, can be modeled via a fluctuating advectiondiffusion equation

$$
\partial_{t} c=-\boldsymbol{u} \cdot \boldsymbol{\nabla} c+\chi_{0} \boldsymbol{\nabla}^{2} c .
$$

where $\chi_{0}$ is the bare (molecular) diffusion coefficient and the advecting velocity $\boldsymbol{u}$ is obtained by convolving the fluid velocity $\boldsymbol{v}$ with a smoothing kernel $\boldsymbol{K}_{\sigma}$ that filters out features below the molecular scale $\sigma$,

$$
\boldsymbol{u}(\boldsymbol{r}, t)=\int \boldsymbol{K}_{\sigma}\left(\boldsymbol{r}, \boldsymbol{r}^{\prime}\right) \boldsymbol{v}\left(\boldsymbol{r}^{\prime}, t\right) d \boldsymbol{r}^{\prime} \equiv\left(\boldsymbol{K}_{\sigma} \star \boldsymbol{v}\right)(\boldsymbol{r}, t),
$$

and preserves the zero-divergence condition, $\boldsymbol{\nabla} \cdot \boldsymbol{u}=0$. Physically, one can think of $\sigma$ as representing the size of the molecular cage in the case of molecular diffusion and the radius of the tracer particles for colloidal diffusion. We stress that the smoothing of the fluctuating velocity field $\boldsymbol{v}$ is necessary to avoid divergence (ultraviolet catastrophe) of the effective diffusion coefficient of the tracer particle obtained below. Thus, the molecular scale details enter (3) in two ways: through the term $\chi_{0} \nabla^{2} c$ and the smoothing of $\boldsymbol{u}$ below scale $\sigma$. As we will see below, the smoothing turns out to be more important for transport than the first. Molecular dynamics simulations have confirmed that (1) and (3) accurately model diffusive mixing between two initially phase-separated fluids down to essentially molecular scales [14]. Note that an additional (mathematically problematic) multiplicative noise term $\boldsymbol{\nabla} \cdot\left(\sqrt{2 \chi_{0} c} \mathcal{W}_{c}\right)$, where $\mathcal{W}_{c}(\boldsymbol{r}, t)$ is a white noise random vector field, needs to be included in (3) to capture equilibrium concentration fluctuations [15]; we do not include this term in this work in order to focus our attention on the nonequilibrium (giant) fluctuations that appear due to the advection by the fluctuating velocity.

In liquids, diffusion of mass is much slower than that of momentum, i.e. the velocity evolves fast compared to the concentration. This separation of time scales is measured by the Schmidt number and it can be used to eliminate the velocity [16, 17]. This procedure, the details of which are presented elsewhere [15], gives a limiting stochastic advection-diffusion equation for the concentration which reads [26]

$$
\begin{aligned}
\partial_{t} c & =-\boldsymbol{w} \odot \boldsymbol{\nabla} c+\chi_{0} \boldsymbol{\nabla}^{2} c \\
& =-\boldsymbol{w} \cdot \boldsymbol{\nabla} c+\chi_{0} \boldsymbol{\nabla}^{2} c+\boldsymbol{\nabla} \cdot[\boldsymbol{\chi}(\boldsymbol{r}) \boldsymbol{\nabla} c]
\end{aligned}
$$

where the first equality shows the equation in Stratonovich's interpretation and the second in Ito's. Here the advection velocity $\boldsymbol{w}(\boldsymbol{r}, t)$ is divergence free $(\nabla$. $\boldsymbol{w}=0)$ and white-in-time, with covariance proportional to a Green-Kubo integral of the auto-correlation function of $\boldsymbol{u}(\boldsymbol{r}, t)$, i.e. $\left\langle\boldsymbol{w}(\boldsymbol{r}, t) \otimes \boldsymbol{w}\left(\boldsymbol{r}^{\prime}, t^{\prime}\right)\right\rangle=\boldsymbol{\mathcal { R }}\left(\boldsymbol{r}, \boldsymbol{r}^{\prime}\right) \delta\left(t-t^{\prime}\right)$ where

$$
\mathcal{R}\left(\boldsymbol{r}, \boldsymbol{r}^{\prime}\right)=2 \int_{0}^{\infty}\left\langle\boldsymbol{u}(\boldsymbol{r}, t) \otimes \boldsymbol{u}\left(\boldsymbol{r}^{\prime}, t+t^{\prime}\right)\right\rangle d t^{\prime},
$$

and the enhancement of the diffusion coefficient is $\chi(\boldsymbol{r})=\frac{1}{2} \mathcal{R}(\boldsymbol{r}, \boldsymbol{r})$. Similar equations, but with a distinct form of the covariance $\mathcal{R}$, appear in the Kraichnan model of turbulent transport [18, 19] (see Sec. 4.1 in [20). It can be shown [15] that at the Lagrangian level (individual tracer trajectories) $(5)$ is equivalent to the well-known equations of Brownian Dynamics with hydrodynamic interactions (correlations) of a form similar to the Rotne-Prager tensor [21], which is widely used as a model for diffusion in dilute colloidal suspensions.

Equation (5) has properties that may seem paradoxical at first sight but have important implications for transport in liquids. Indeed notice that it is easy to take the average of this equation in Ito's form to deduce that the ensemble average of the concentration obeys Fick's law,

$$
\partial_{t}\langle c\rangle=\boldsymbol{\nabla} \cdot\left(\boldsymbol{\chi}_{\mathrm{eff}} \boldsymbol{\nabla}\langle c\rangle\right) \text { where } \boldsymbol{\chi}_{\mathrm{eff}}=\chi_{0} \boldsymbol{I}+\boldsymbol{\chi},
$$

which is a well-known result that can be justified rigorously (c.f. Eq. (255) in [17]) and holds even in the absence of bare diffusion, $\chi_{0}=0$. This is surprising considering that (5) is time-reversible when $\chi_{0}=0$, as made clear by Stratonovich's form of this equation. Furthermore, the same equation (7) holds for all moments of $c$ when $\chi_{0}=0$. This is no contradiction: the "dissipative" term $\boldsymbol{\nabla} \cdot[\boldsymbol{\chi}(\boldsymbol{r}) \boldsymbol{\nabla} c]$ and the stochastic forcing term $-\boldsymbol{w} \cdot \boldsymbol{\nabla} c$ are signatures of the same physical process, advection by thermal velocity fluctuations. Including the first term but omitting the second violates fluctuationdissipation balance and cannot be justified. For example, the stochastic terms in (5) need to be retained to obtain the giant fluctuations seen in a particular instance (realization) of the diffusive mixing process.

Next we estimate the diffusion enhancement, $\boldsymbol{\chi}(\boldsymbol{r})=$ $\frac{1}{2} \mathcal{R}(\boldsymbol{r}, \boldsymbol{r})$, to get an intuitive understanding of its role. Since $\boldsymbol{v}(\boldsymbol{r}, t)$ solves the linearized fluctuating NavierStokes equation (1), it is not hard to show that

$$
\int_{0}^{\infty}\left\langle\boldsymbol{v}(\boldsymbol{r}, t) \otimes \boldsymbol{v}\left(\boldsymbol{r}^{\prime}, t+t^{\prime}\right)\right\rangle d t^{\prime}=\frac{k_{B} T}{\eta} \boldsymbol{G}\left(\boldsymbol{r}, \boldsymbol{r}^{\prime}\right),
$$

where $\boldsymbol{G}$ is the Green's function (Oseen tensor) for the steady Stokes equation with unit viscosity, $\boldsymbol{\nabla} \pi=\boldsymbol{\nabla}^{2} \boldsymbol{v}+$ $\boldsymbol{f}$ subject to $\boldsymbol{\nabla} \cdot \boldsymbol{v}=0$ and appropriate boundary conditions. Inserting this expression in (6) implies that

$$
\mathcal{R}\left(\boldsymbol{r}, \boldsymbol{r}^{\prime}\right)=\left(\boldsymbol{K}_{\sigma} \star \boldsymbol{G} \star \boldsymbol{K}_{\sigma}^{T}\right)\left(\boldsymbol{r}, \boldsymbol{r}^{\prime}\right)
$$

To proceed, recall that for an infinite isotropic system, $\boldsymbol{G}\left(\boldsymbol{r}, \boldsymbol{r}^{\prime}\right) \equiv \boldsymbol{G}\left(\boldsymbol{r}-\boldsymbol{r}^{\prime}\right)$ is the Oseen tensor, the Fourier tranform of which reads $\widehat{\boldsymbol{G}}_{\boldsymbol{k}}=k^{-2}\left(\boldsymbol{I}-k^{-2} \boldsymbol{k} \otimes \boldsymbol{k}\right)$. Let 
us employ an isotropic filtering kernel $\boldsymbol{K}_{\sigma}$ that cuts off the fluctuations in the advective velocity $\boldsymbol{w}$ at both large and small scales to account respectively for the finite extent of the system $L$ and the filtering at the molecular scale $\sigma$, and assume that the Fourier transform of $\boldsymbol{R}\left(\boldsymbol{r}-\boldsymbol{r}^{\prime}\right) \equiv \boldsymbol{R}\left(\boldsymbol{r}, \boldsymbol{r}^{\prime}\right)$ is

$$
\hat{\mathcal{R}}_{\boldsymbol{k}}=\frac{2 k_{B} T}{\eta} \frac{k^{2} L^{4}}{\left(1+k^{4} L^{4}\right)\left(1+k^{2} \sigma^{2}\right)}\left(\boldsymbol{I}-\frac{\boldsymbol{k} \otimes \boldsymbol{k}}{k^{2}}\right)
$$

Converting 10 to real space gives an isotropic enhancement to the diffusion tensor $\chi=\mathcal{R}(0) / 2=$ $(2 \pi)^{-d} \int\left(\hat{\boldsymbol{R}}_{\boldsymbol{k}} / 2\right) d \boldsymbol{k}=\chi \boldsymbol{I}$. This Fourier integral is exactly the one that appears in the linearized steadystate (static) approximate renormalization theory when $\nu \gg \chi_{0}[7]$. Here we obtain the same result with a simple, general, and precise calculation that gives [27] for $L \gg \sigma$

$$
\chi \sim \frac{k_{B} T}{\eta} \begin{cases}(4 \pi)^{-1} \ln (L / \sigma) & \text { if } d=2 \\ (6 \pi \sigma)^{-1} & \text { if } d=3 .\end{cases}
$$

In three dimensions (11) gives the Stokes-Einstein prediction $\chi \sim \chi_{S E}=k_{B} T /(6 \pi \eta \sigma)$ for the diffusion coefficient of a slowly-diffusing no-slip rigid sphere of radius $\sigma$. In two dimensions, the effective diffusion coefficient grows logarithmically with system size, in agreement with the Einstein relation and the Stokes paradox for the mobility of a disk of radius $\sigma$. This system-size dependence of the effective diffusion coefficient has been verified using steady-state particle simulations [9, 22, Note also that (11) allows us to validate a posteriori the assumption of large separation of time scales between concentration and momentum diffusion. Specifically, the limiting equation (5) is a good approximation to (3) if the effective Schmidt number $\mathrm{Sc}=\nu / \chi_{\mathrm{eff}}=\nu /\left(\chi_{0}+\chi\right) \gg 1$. This is indeed the case in practice for simple liquids and macromolecular solutions.

The measured diffusion coefficients in molecular liquids and macromolecular solutions closely match the StokesEinstein prediction. This suggests that in realistic fluids diffusive transport is dominated by advection by the velocity fluctuations, $\chi \gg \chi_{0}$. Since we know that each realization follows a strictly reversible dynamics when $\chi_{0}=0$, but that the evolution of the mean is dissipative even in this case since $\chi_{\text {eff }}=\chi>0$, it is important to understand the difference in the behavior of the ensemble mean of the diffusive mixing process, described by (7), and the behavior of an individual realization, described by (5).

To this end, we resort to numerical experiments using finite-volume [23] Eulerian methods [28], as well as Lagrangian tracers algorithms 29] that we have developed specifically for the purpose of simulating the limiting dynamics (5) at $\chi_{0}>0$ and $\chi_{0}=0$, respectively. Details of these multiscale numerical methods are given elsewhere [15]. Let us consider the temporal decay of a smooth single-mode initial perturbation

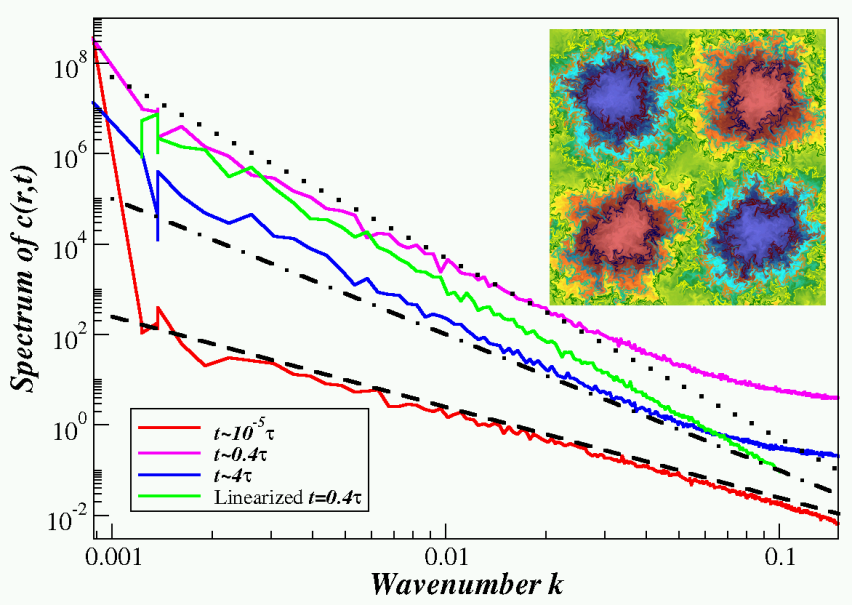

FIG. 1: The decay of a single-mode initial condition. The inset shows the concentration at a time $t \approx 2 \tau$, as obtained from a Lagrangian simulation with $2048^{2}$ tracers and no bare diffusion, along with numerical approximations to the contour lines. The main figure shows the power spectrum of an individual realization of the concentration $c(\boldsymbol{r}, t)$ at several times, as obtained using an Eulerian algorithm for solving (5). The power of individual modes $\boldsymbol{k}$ with nearby $k$ is averaged and the result is shown with colored solid lines, while dashed/dotted lines show power laws $k^{-2}, k^{-3}$ and $k^{-4}$ for comparison. At early times $t \ll \tau=\left(2 \chi_{\text {eff }} k_{0}^{2}\right)^{-1}$ (red line) power is being transferred from mode $\boldsymbol{k}_{0} \approx 2 \pi / L \approx 10^{-3}$, initially excited to have spectral power $p_{\boldsymbol{k}_{0}} \approx 7 \cdot 10^{8}$, to the rest of the modes, leading to a spectrum $\sim k^{-2}$. At late times $t \gtrsim \tau$ (magenta and blue lines), a steadily-decaying shape of the spectrum is reached where power transferred from the larger scales is dissipated at the small scales via bare diffusion. Numerically linearized fluctuating hydrodynamics predicts a spectrum $\sim k^{-4}$ (green line) 3.

$c(\boldsymbol{r}, 0)=\sin (2 \pi x / L) \sin (2 \pi y / L)$ in two dimensions. The ensemble mean $\langle c\rangle$ follows the simple diffusion equation (7), and therefore remains a single-mode field with an amplitude decaying as $\exp (-t / \tau)$, where $\tau=\left(2 \chi_{\mathrm{eff}} k_{0}^{2}\right)^{-1}$ is a decay time and $k_{0}$ is the initially excited wavenumber. In the inset of Fig. 1, we show a single instance (realization) of the concentration at time $t \approx 2 \tau$ when $\chi_{0}=0$. The figure reveals characteristic giant (longranged) fluctuations in particular realizations of the diffusive process, with the contour lines of the concentration becoming rougher as time progresses [30. These enhanced nonequilibrium fluctuations stem from the development of a power-law spectrum as the mixing progresses, as predicted by linearized fluctuating hydrodynamics 3 . The evolution of the power spectrum during the diffusive decay is illustrated in Fig. 1.

The conserved quantity $\int\left(c^{2} / 2\right) d \boldsymbol{r}$ injected via the initial perturbation away from equilibrium is effectively dissipated through a mechanism similar to the energy cascade observed in turbulent flows. Advection transfers power from the large length scales to the small length 
scales, effectively dissipating the power injected into the large scales via the initial condition. A straightforward calculation that is detailed elsewhere [15] shows that the total rate at which power is lost ("dissipated") from mode $\boldsymbol{k}_{0}$ is given by $\boldsymbol{k}_{0} \cdot \boldsymbol{\chi} \cdot \boldsymbol{k}_{0}$. This is exactly the same rate of dissipation as one would get for ordinary diffusion with diffusion tensor $\chi$. However, in simple diffusion all other modes would remain unexcited and there would be no giant fluctuations.

At late times of the diffusive decay, $t \sim \tau$, one expects that a self-similar state will be reached in which the shape of the spectrum of $c$ does not change as it decays exponentially in time as $\exp (-t / \tau)$. This is indeed what we observe, and the shape of the decaying spectrum is shown in Fig. 11. Numerically we observe that most of the bare dissipation occurs at the largest wavenumbers. Note however that the shape of the spectrum at the large wavenumbers is strongly affected by discretization artifacts and the presence of (small) bare diffusion. These numerical grid artifacts can be eliminated by using the Lagrangian tracer algorithm, which leads to a similar power-law behavior [15].

In the literature, linearized fluctuating hydrodynamics is frequently used to obtain the steady-state spectrum of fluctuations [3. In the limit of large Schmidt numbers, the standard heuristic approach leads to the additivenoise equation,

$$
\partial_{t} \tilde{c}=-\boldsymbol{w} \cdot \boldsymbol{\nabla}\langle c\rangle+\nabla \cdot\left[\chi_{\mathrm{eff}} \boldsymbol{\nabla} \tilde{c}\right]
$$

where $\langle c\rangle$ is the ensemble mean, which follows (7). Note that we have not accounted for equilibrium concentration fluctuations in 12 since our focus here is on the nonequilibrium fluctuations and we wish to more accurately measure the power-law spectrum. Equation 12 can easily be solved analytically in the Fourier domain when $\boldsymbol{\nabla}\langle c\rangle=\boldsymbol{h}$ is a weak externally applied constant gradient to obtain a spectrum $\left(\boldsymbol{h} \cdot \hat{\chi}_{\boldsymbol{k}} \cdot \boldsymbol{h}\right) /\left(\chi_{\mathrm{eff}} k^{2}\right) \sim k^{-4}$ for intermediate wavenumbers. For finite gradients and more realistic boundary conditions, we can solve 12 numerically with the same algorithm used to solve the full nonlinear equation (5) by simply reducing the magnitude of the fluctuations by a large factor and then increasing the spectrum of the fluctuations by the same factor to obtain the spectrum of $\tilde{c}$ [23]. The result of this numerically-linearized calculation for the single-mode initial condition is shown in Fig. 1 and seen to follow the expected $k^{-4}$ powerlaw [3. This power law is not in a very good agreement with the spectrum obtained by solving the full nonlinear equation (5), which appears closer to $k^{-3}$ in the twodimensional setting we study here.

If there were only random advection, with no bare diffusion, the transfer of energy from the coarse to the fine scales would continue indefinitely, since the dynamics is reversible and there is nothing to dissipate the power. However, any features in $c$ at length scales below molecular scales have no clear physical meaning. In fact, con- tinuum models are inapplicable at those scales. In typical experiments, such as FRAP measurements of diffusion coefficients, one observes the concentration spatiallycoarse grained at scales much larger than the molecular scale. It is expected that not resolving (coarse-graining) the microscopic scales will lead to true dissipation and irreversibility in the coarse-grained dynamics. Such coarsegraining can take form of ensemble averaging, or elimination of slow degrees of freedom. In either case, the loss of knowledge about the small scales will lead to positive entropy production.

It is reasonable to expect that one can replace the molecular scale details, or even all details of the dynamics at scales below some mesoscopic observation scale $\delta$, by effective dissipation. In particular, we suggest that small-scale details in (5) can be replaced by a diffusive term with suitably chosen renormalized bare coefficient. This renormalization needs to be carried in such a way that the effective diffusion coefficient in the equation for the mean remains equal to $\chi_{\text {eff }}=\chi_{0} \boldsymbol{I}+\chi$. A partial ensemble averaging of (5) can be used to achieve this goal [15], and this calculation leads to a spatially coarsegrained model for diffusion in liquids,

$$
\partial_{t} c_{\delta}=-\boldsymbol{w}_{\delta} \odot \boldsymbol{\nabla} c_{\delta}+\boldsymbol{\nabla} \cdot\left[\left(\chi_{0} \boldsymbol{I}+\Delta \boldsymbol{\chi}_{\delta}\right) \boldsymbol{\nabla} c_{\delta}\right],
$$

where $c_{\delta}=\boldsymbol{K}_{\delta} \star c$ denotes the concentration filtered at the mesoscopic scale $\delta$, the white-in-time random velocity $\boldsymbol{w}_{\delta}$ has covariance $\boldsymbol{K}_{\delta} \star \boldsymbol{R} \star \boldsymbol{K}_{\delta}^{T}$, and $\chi_{0}$ is renormalized by

$$
\Delta \boldsymbol{\chi}_{\delta}(\boldsymbol{r})=\frac{1}{2}\left(\mathcal{R}-\boldsymbol{K}_{\delta} \star \mathcal{R} \star \boldsymbol{K}_{\delta}^{T}\right)(\boldsymbol{r}, \boldsymbol{r})
$$

In Ito's form (13) is the same as (5) with $\boldsymbol{w}$ replaced by $\boldsymbol{w}_{\delta}$. Note that the renormalized bare diffusion coefficient $\chi_{0}(\delta)=\chi_{0} \boldsymbol{I}+\Delta \chi_{\delta}$ in (13) is nonzero even if $\chi_{0}=$ 0 . This true dissipation is a remnant of the unresolved (eliminated) small scales. However, it is important to stress that $\chi_{0}(\delta)$ is not a material constant, but rather, depends on the mesoscopic lengthscale $\delta$.

To test (13), consider diffusive mixing between two initially phase-separated fluids in two dimensions with periodic boundary conditions. We start with concentration $c=1$ in a thin horizontal stripe, $c=0$ everywhere. This could, for example, model a stripe in a FRAP experiment in which a laser beam combined with a diffraction grating is used to create a striped pattern of fluorescently labeled tracers at $t=0$. In Fig. 2 we show snapshots of the concentration field at a later time, for $\delta=0$ (no coarsegraining) in the top panel, and $\delta=3 \sigma$ in the middle and bottom panels. Specifically, in the middle panel we show the spatially smoothed concentration $\boldsymbol{K}_{\delta} \star c$. For comparison, in the bottom panel we show an instance of the solution of the proposed coarse-grained diffusion equation (13). Since $\boldsymbol{u}$ and $\boldsymbol{w}$ are spatially-smooth velocity fields, advection by these fields leads to behavior qualitatively different from diffusion when $\chi_{0}=0$. Specifically, if the initial concentration $c(\boldsymbol{r}, 0)$ has a sharp interface, this interface will remain sharp at all times, even if it becomes 

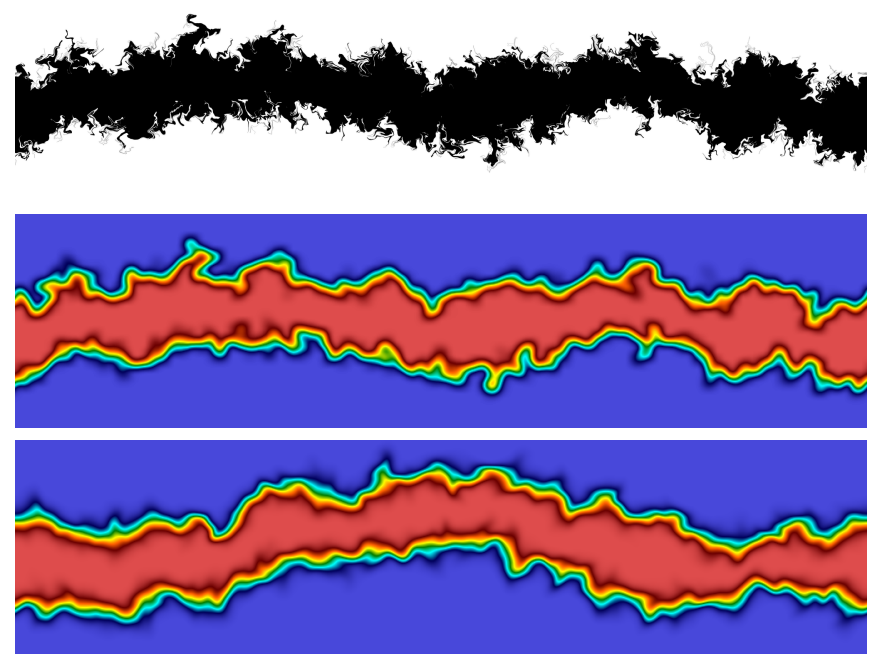

FIG. 2: Top panel: A snapshot of the concentration $c$ for diffusive mixing of two miscible fluids in the absence of bare diffusion, starting from concentration being unity (black) in a horizontal stripe occupying one third of the periodic domain, and zero (white) elsewhere. The top and bottom interface are represented with about half a million Lagrangian tracers each. Middle panel: The spatially-coarse grained concentration $c_{\delta}$ obtained by blurring the top panel using a Gaussian filter with standard deviation $\delta=3 \sigma$. Bottom panel: An independent snapshot of the spatially coarse-grained concentration $c_{\delta}$ at the same point in time as the top panel, obtained by solving (13) with an Eulerian method using a grid of $2048 \times 512$ finitevolume cells. A Gaussian filter of width $\delta=3 \sigma$ is used to filter the discrete velocity. The effective diffusion coefficient $\chi_{\text {eff }}$ is the same as in the top panel.

very rough, at all times, in every realization. Therefore, the top panel in the figure is black and white. In the presence of true (bare) dissipation, the interface between the two fluids does not remain sharp, and a range of concentrations $0 \leq c \leq 1$ appears for $t>0$. Therefore, the middle and bottom panels in the figure show a spectrum of colors.

In large three dimensional systems, when the spatial coarse-graining is performed at macroscopic scales $\delta \gg \sigma$, it is expected that 13 will converge in some sense to the linearized fluctuating hydrodynamics equation (12, as suggested by renormalization arguments [7]. While we are not aware of mathematical tools to prove this type of statement, a plausible argument goes as follows. In three dimensions, as $\delta \rightarrow \infty$, the renormalization of the diffusion coefficient approaches the Stokes-Einstein value, $\Delta \boldsymbol{\chi}_{\delta} \rightarrow \boldsymbol{\chi}$, and the stochastic term $\boldsymbol{w}_{\delta} \odot \boldsymbol{\nabla} c_{\delta}$ becomes negligible because most of the power (spectral intensity) in the the random advection velocity $\boldsymbol{w}_{\delta}$ is removed by the filtering (since the spectrum of $\boldsymbol{w}$ decays like $k^{-2}$, in three dimensions the power distribution is independent of $k$ ). Therefore the noise will become "weak" in a suitable sense and the fluctuations can be linearized around the mean. This is not true in two dimensions, where large scale features in $\boldsymbol{w}_{\delta}$ give the dominant contribution to the effective diffusion and contain the majority of the spectral power of $\boldsymbol{w}$ (in two dimensions the power distribution decays like $k^{-1}$ ). Therefore, linearization is certainly not appropriate in two dimensions even if $\delta \gg \sigma$. Thin films may exhibit an intermediate behavior depending on the scale of observation relative to the thickness of the thin film [24].

Summing up, in both two and three dimensions the behavior of mixing processes in liquids cannot be described by Fick's law at mesoscopic scales. One must include random advection by the mesoscopic scales of the velocity fluctuations in order to reproduce not just the behavior of the mean but also the long-range correlated fluctuations observed in individual realizations. This emphasizes the crucial distinction between the selfdiffusion of individual tracers and the collective diffusion of many hydrodynamically-correlated tracers. The traditional Fick's diffusion constant is only meaningful under special conditions (e.g., large three-dimensional bulk systems observed at macroscopic scales) which may not in fact be satisfied in experiments aimed to measure "the" diffusion coefficient. A length scale of observation (coarse-graining) must be attached to the diffusion coefficient value in order to make it a "material constant" that can be used in a predictive model of diffusive transport [9]. Furthermore, the measured diffusion coefficient is strongly affected by boundary conditions (confinement) [10, 22, 24].

We hope that these results will spur interest in designing experiments that carefully examine diffusion at a broad range of length scales. Existing experiments have been able to measure concentration fluctuations across a wide range of lenghtscales transverse to the gradient, but fluctuations are averaged longitudinally over essentially macroscopic scales (thickness of the sample) [4 6 ]. While FRAP experiments routinely look at diffusion at micrometer scales, we are not aware of any work that has even attempted to account for the effect of thermal fluctuations. Giant fluctuations are expected to be more easily observed in thin liquid films due to the quasitwo dimensional geometry [24, 25]. In the future we will consider extensions of our approach to multispecies liquid mixtures. Such extensions are expected to lead to a better understanding of the physics of diffusion in fluid mixtures, including a generalized Stokes-Einstein relation for inter-diffusion coefficients in dilute multispecies solutions. 
We would like to acknowledge Florencio Balboa Usabiaga and Andreas Klockner for their help in developing a GPU implementation of the numerical methods, and Leslie Greengard for advice on the non-uniform FFT algorithm. We are grateful to Alberto Vailati, Alejandro Garcia, John Bell, Sascha Hilgenfeldt, Mike Cates and Ranojoy Adhikari for their insightful comments. A. D. was supported in part by the NSF grant DMS1115341 and the DOE Early Career award de-sc0008271. T. F.was supported in part by the DOE CSGF grant DE-FG02-97ER25308. E. V.-E. was supported by the DOE ASCR grant DE-FG02-88ER25053, the NSF grant DMS07-08140, and the ONR grant N00014-11-1-0345.

[1] J. R. Dorfman, T. R. Kirkpatrick, and J. V. Sengers, Annual Review of Physical Chemistry 45, 213 (1994), ISSN 0066-426X.

[2] D. Brogioli, A. Vailati, and M. Giglio, Phys. Rev. E 61, R1 (2000).

[3] J. M. O. D. Zarate and J. V. Sengers, Hydrodynamic fluctuations in fluids and fluid mixtures (Elsevier Science Ltd, 2006).

[4] A. Vailati and M. Giglio, Nature 390, 262 (1997).

[5] F. Croccolo, D. Brogioli, A. Vailati, M. Giglio, and D. S. Cannell, Phys. Rev. E 76, 041112 (2007).

[6] A. Vailati, R. Cerbino, S. Mazzoni, C. J. Takacs, D. S. Cannell, and M. Giglio, Nature Communications 2, 290 (2011).

[7] D. Bedeaux and P. Mazur, Physica 73, 431 (1974).

[8] D. Brogioli and A. Vailati, Phys. Rev. E 63, 12105 (2000).

[9] A. Donev, A. L. Garcia, A. de la Fuente, and J. B. Bell, J. of Statistical Mechanics: Theory and Experiment 2011, P06014 (2011).

[10] F. Detcheverry and L. Bocquet, Phys. Rev. Lett. 109, 024501 (2012).

[11] P. Mazur and D. Bedeaux, Physica 75, 79 (1974).

[12] D. Bedeaux and P. Mazur, Physica A Statistical Mechanics and its Applications 80, 189 (1975).

[13] J. T. Hynes, R. Kapral, and M. Weinberg, J. Chem. Phys. 70, 1456 (1979).

[14] A. Donev, A. J. Nonaka, Y. Sun, T. G. Fai, A. L. Garcia, and J. B. Bell (2014), to appear in CAMCOS, ArXiv preprint 1212.2644 .

[15] A. Donev, T. G. Fai, and E. Vanden-Eijnden (2014), to appear in JSTAT, ArXiv preprint 1312.1894.
[16] G. C. Papanicolaou, Rocky Mountain J. Math 6, 653 (1976).

[17] A. Majda, I. Timofeyev, and E. Vanden-Eijnden, Nonlinearity 19, 769 (2006).

[18] G. L. Eyink and J. Xin, J. Stat. Phys. 100, 679 (2000).

[19] M. Chaves, G. Eyink, U. Frisch, and M. Vergassola, Phys. Rev. Lett. 86, 2305 (2001).

[20] A. J. Majda and P. R. Kramer, Physics Reports 314, 237 (1999), ISSN 0370-1573.

[21] S. Delong, F. B. Usabiaga, R. Delgado-Buscalioni, B. E. Griffith, and A. Donev (2014), in press, J. Chem. Phys.

[22] A. Donev, A. L. Garcia, A. de la Fuente, and J. B. Bell, Phys. Rev. Lett. 106, 204501 (2011).

[23] F. B. Usabiaga, J. B. Bell, R. Delgado-Buscalioni, A. Donev, T. G. Fai, B. E. Griffith, and C. S. Peskin, SIAM J. Multiscale Modeling and Simulation 10, 1369 (2012).

[24] J. Bechhoefer, J.-C. Géminard, L. Bocquet, and P. Oswald, Phys. Rev. Lett. 79, 4922 (1997).

[25] B. Schulz, D. Täuber, F. Friedriszik, H. Graaf, J. Schuster, and C. Von Borczyskowski, Physical Chemistry Chemical Physics 12, 11555 (2010).

[26] Here $\boldsymbol{w} \odot \boldsymbol{\nabla} c$ and $\boldsymbol{w} \cdot \boldsymbol{\nabla} c$ are short-hand notations for $\sum_{k}\left(\phi_{k} \cdot \nabla c\right) \circ d \mathcal{B}_{k} / d t$ and $\sum_{k}\left(\phi_{k} \cdot \nabla c\right) d \mathcal{B}_{k} / d t$, respectively, where $\mathcal{B}_{k}(t)$ are independent Wiener processes and $\phi_{k}$ are basis functions such that $\mathcal{R}\left(\boldsymbol{r}, \boldsymbol{r}^{\prime}\right)=$ $\sum_{k} \phi_{k}(\boldsymbol{r}) \otimes \phi_{k}\left(\boldsymbol{r}^{\prime}\right)$.

[27] Some of the coefficients in 11 depend on the exact form of the spectrum $\hat{\mathcal{R}}_{\boldsymbol{k}}$ in 10 .

[28] We checked that the results of these simulations compare well to those obtained by integrating the resolved dynamics 1] 3] with $S_{c}=\nu / \chi_{\text {eff }} \sim 10^{3}-10^{4}[23$. The overdamped simulations can reach the same time scales in much less (by a factor of about $S_{c}$ ) computational effort than the direct numerical simulation because they bypass the need to resolve the fast velocity fluctuations.

[29] The Lagrangian description associated with (5) reads

$$
d \boldsymbol{q}=\sum_{k} \phi_{k}(\boldsymbol{q}) \circ d \mathcal{B}_{k}+\sqrt{2 \chi_{0}} d \mathcal{B}_{\boldsymbol{q}}
$$

where a single realization of the random field $\sum_{k} \phi_{k} \circ d \mathcal{B}_{k}$ advects all of the walkers. This induces correlations between their trajectories which crucially affect the physics of the collective diffusion of the tracers.

[30] We have performed hard-disk molecular dynamics simulation of this mixing process and observed the same qualitative behavior seen in the inset of Fig. 1 . 